\title{
Glossary of East Asian Names
}

Bai Shouyi 白壽彝

Bao Tingliang 保廷樑

Cai Dayu 蔡大愚

Chen Guangyuan 陳廣元

Chen Keli 陳克禮

Chen Si 陳思

Da Pusheng 達浦生

Ding Baochen 丁寶臣

Ding Shiren 丁士仁

Ding Zhuyuan 丁竹園

Du Wenxiu 杜文秀

Fei Xiaotong 費孝通

Feng Zenglie 馮增烈

Ha Decheng 哈徳成

Hai Weiliang 海維諒

Han Haichao 韓海朝

$\mathrm{He}$ Yaozu 賀耀祖

Hong Lu 洪爐

Hu Dengzhou 胡登洲

Hu Fangquan 胡枋權

Hu Songshan 虎嵩山 
Huang Wanjun 黄万均

Huang Zhenpan 黃鎮磐

Jiang Jieshi (Chiang Kai-shek) 蔣介石

Jin Diangui 金殿桂

Jin Jitang 金吉堂

Jing Rizhen 景日眕

Kang Youxi 康有雪

Kawamura Kyōdō 川村狂堂

Li Xizhen 李希真

Li Zhenzhong 李振中

Liang Qichao 梁啓超

Liang Yijun 梁以浚

Lin Changxing 林昌興

Lin Song 林松

Lin Xinghua 林興華

Lin Zhongming 林仲明

Liu Mengyang 劉孟揚

Liu Sanjie 劉三杰

Liu Zhi 劉智

Lu Jiuyuan (Xiangshan) 陸九淵 (象山)

Lu You 鹿祐

Ma Anli 馬安禮

Ma Anliang 馬安良

Ma Dexin (Fuchu) 馬德新 (復初)

Ma Enxin 馬恩信

Ma Fuxiang 馬福祥

Ma Jian 馬堅

Ma Lianyuan 馬聯元

Ma Linyi 馬鄰翼

Ma Mingliang 馬明良

Ma Qiang 馬強

Ma Qixi 馬啟西

Ma Songting 馬松亭

Ma Tong 馬通

Ma Wanfu 馬萬福 
Ma Xian 馬賢

Ma Xingzhou 馬興周

Ma Xiulan 馬秀蘭

Ma Yuming (pseud. Xiao Ma A-ge) 馬玉明 (小馬 阿哥)

Ma Zhixin (Baha Ahong) 馬志信

Ma Zhu 馬注

Min Shengguang 敏生光

$\mathrm{Na}$ Zhong 納忠

Pang Shiqian 龐士謙

Qi Xueyi 祁學義

Sai Dianchi (Sayyid Ajall Shams al-Din) 賽典赤

Sakuma Teijirō 佐久間貞次郎

Sha Shanyu (Shouyu) 沙善余 (守愚)

Shan Guoqing 山國慶

Shi Zizhou 時子周

Shou Jin 壽金

Sun Yat-sen (Sun Yixian, Sun Zhongshan) 孫逸仙, 孫中山

Tanaka Ippei 田中逸平

Tang Yichen 唐易塵

Tong Jili 童基立

Wang Daiyu 王岱鲯

Wang Enrong 王恩栄

Wang Jingzhai 王静新

Wang Jixian 王繼賢

Wang Kuan 王寬

Wang Mengyang 王夢揚

Wang Ruilan 王瑞蘭

Wang Shiming 王世明

Wang Yingqi 王英麒

Wang Yousan 王友三

Wu Sangui 吳三桂

Wu Zixian 伍子先

Wuhuaguo (pseud.) 無花果

$\mathrm{Xu}$ Yuanzheng 徐元正

Xue Wenbo 薛文波 
Yang Du 楊度

Yang Feilu 楊斐菉

Yang Huaizhong 楊懷中

Yang Sishi 仰思室

Yin Boqing 尹伯清

Yu Ke 愚克

Yu Zhengui 余振貴

Yuan Guozuo 袁國做

Yuan Ruqi 袁汝琦

Yuan Shikai 袁世凱

Zhang Bingduo 張秉鐸

Zhang Chengqian 張承遷

Zhang Weizhen 張維真

Zhang Zai 張載

Zhao Zhongqi 趙鍾奇

Zhou Dunyi 周敦頣

Zhou Lianggong 周亮工 